\title{
Positive solutions for a class of fractional 3-point boundary value problems at resonance
}

\author{
Yongqing Wang ${ }^{1,2^{*}}$ (1) and Lishan Liu ${ }^{2,3}$
}

\section{"Correspondence:}

wyqing9801@163.com

'School of Statistics, Qufu Normal

University, Qufu, Shandong 273165 ,

P.R. China

${ }^{2}$ School of Mathematical Sciences,

Qufu Normal University, Qufu,

Shandong 273165, P.R. China

Full list of author information is

available at the end of the article

\section{Abstract}

In this paper, we study the nonlocal fractional differential equation:

$$
\left\{\begin{array}{l}
D_{0+}^{\alpha} u(t)+f(t, u(t))=0, \quad 0<t<1 \\
u(0)=0, \quad u(1)=\eta u(\xi)
\end{array}\right.
$$

where $1<\alpha<2,0<\xi<1, \eta \xi^{\alpha-1}=1, D_{0+}^{\alpha}$ is the standard Riemann-Liouville derivative, $f:[0,1] \times[0,+\infty) \rightarrow \mathbb{R}$ is continuous. The existence and uniqueness of positive solutions are obtained by means of the fixed point index theory and iterative technique.

Keywords: fractional differential equation; positive solution; resonance; fixed point index

\section{Introduction}

In this paper, we consider the following fractional differential equation:

$$
\left\{\begin{array}{l}
D_{0+}^{\alpha} u(t)+f(t, u(t))=0, \quad 0<t<1, \\
u(0)=0, \quad u(1)=\eta u(\xi),
\end{array}\right.
$$

where $1<\alpha<2,0<\xi<1, \eta \xi^{\alpha-1}=1, D_{0+}^{\alpha}$ is the standard Riemann-Liouville derivative, $f:[0,1] \times[0,+\infty) \rightarrow \mathbb{R}$ is continuous. Problem (1.1) happens to be at resonance, since $\lambda=0$ is an eigenvalue of the linear problem

$$
\left\{\begin{array}{l}
-D_{0+}^{\alpha} u=\lambda u, \quad 0<t<1, \\
u(0)=0, \quad u(1)=\eta u(\xi),
\end{array}\right.
$$

and $c t^{\alpha-1}, c \in \mathbb{R}$, is the corresponding eigenfunction.

Fractional differential equations occur frequently in various fields such as physics, chemistry, engineering and control of dynamical systems, etc. During the last few decades, many papers and books on fractional calculus and fractional differential equations have appeared (see [1-22] and the references therein).

(c) The Author(s) 2017. This article is distributed under the terms of the Creative Commons Attribution 4.0 International License (http://creativecommons.org/licenses/by/4.0/), which permits unrestricted use, distribution, and reproduction in any medium, provided you give appropriate credit to the original author(s) and the source, provide a link to the Creative Commons license, and indicate if changes were made. 
When $0<\eta \xi^{\alpha-1}<1$, problem (1.1) is non-resonant. In [9], the author studied the existence of positive solutions for the non-resonant case by means of the fixed point index theory under sublinear conditions.

In [18], the authors investigated the existence and multiplicity results of positive solutions by using of the fixed point theorem for the fractional differential equation given by

$$
\left\{\begin{array}{l}
D_{0+}^{\alpha} u(t)=f(t, u(t)), \quad 0<t<1, \\
u(0)=0, \quad D_{0+}^{\beta} u(1)=a D_{0+}^{\beta} u(\xi),
\end{array}\right.
$$

where $1<\alpha \leq 2,0 \leq \beta \leq 1,0<\xi<1,0 \leq a \leq 1$ with $a \xi^{\alpha-\beta-2}<1-\beta, 0 \leq \alpha-\beta-1$.

Recently, there are some papers dealing with the existence of solutions of fractional boundary value problem at resonance by using the coincidence degree theory due to Mawhin (see [19-22]). In [22], the authors investigated the following fractional threepoint boundary value problem (BVP for short) at resonance:

$$
\left\{\begin{array}{l}
D_{0+}^{\alpha} u(t)+f\left(t, u(t), D_{0+}^{\alpha-1} u(t)\right)=0, \quad 0<t<1 \\
u(0)=0, \quad u(1)=\eta u(\xi)
\end{array}\right.
$$

where $1<\alpha \leq 2,0<\xi<1, \eta \xi^{\alpha-1}=1, D_{0+}^{\alpha}$ is the standard Riemann-Liouville derivative, $f:[0,1] \times \mathbb{R}^{2} \rightarrow \mathbb{R}$ is continuous. By using the coincidence degree theory, the existence of solutions for BVP (1.4) are obtained under certain growth conditions.

To the best of our knowledge, there are only very few papers dealing with the existence of positive solutions for resonant boundary value problems since the corresponding linear operator is non-reversible. For the case that $\alpha$ is an integer, some work has been done dealing with the existence of positive solutions for resonant boundary value problems by using Leggett-Williams norm-type theorem for coincidence (see [23-25]). Webb [26] established existence of positive solutions for second order boundary value problems at resonance by considering equivalent non-resonant perturbed problems with the same boundary conditions.

Inspired by the work mentioned above, in this paper we aim to establish the existence of positive solutions for resonant problem (1.1). The paper is organized as follows. Firstly, we reduce non-perturbed boundary value problems at resonance to equivalent non-resonant perturbed problems with the same boundary conditions. Then we derive the corresponding Green's function and argue its properties. Finally, the existence and uniqueness results of positive solutions are obtained by using of the fixed point index and iterative technique.

\section{Basic definitions and preliminaries}

In this section, we present some preliminaries and lemmas. The definitions and properties of fractional derivative can be found in the literature [1-22].

Definition 2.1 The fractional integral of order $\alpha>0$ of a function $u:(0,+\infty) \rightarrow R$ is given by

$$
I_{0+}^{\alpha} u(t)=\frac{1}{\Gamma(\alpha)} \int_{0}^{t}(t-s)^{\alpha-1} u(s) d s
$$

provided that the right-hand side is point-wise defined on $(0,+\infty)$. 
Definition 2.2 The Riemann-Liouville fractional derivative of order $\alpha>0$ of a function $u:(0,+\infty) \rightarrow R$ is given by

$$
D_{0+}^{\alpha} u(t)=\frac{1}{\Gamma(n-\alpha)}\left(\frac{d}{d t}\right)^{n} \int_{0}^{t}(t-s)^{n-\alpha-1} u(s) d s,
$$

where $n=[\alpha]+1,[\alpha]$ denotes the integer part of number $\alpha$, provided that the right-hand side is point-wise defined on $(0,+\infty)$.

Denote

$$
g(t)=\frac{\alpha-2}{\Gamma(\alpha-1)}+\sum_{k=1}^{+\infty} \frac{t^{k}}{\Gamma((k+1) \alpha-2)} .
$$

It is easy to check that $g^{\prime}(t)>0$ on $(0,+\infty)$, and

$$
g(0)=\frac{\alpha-2}{\Gamma(\alpha-1)}<0, \quad \lim _{t \rightarrow+\infty} g(t)=+\infty .
$$

Therefore, there exists a unique $b^{*}>0$ such that

$$
g\left(b^{*}\right)=0 .
$$

For the convenience in presentation, we here list the assumptions to be used throughout the paper.

$\left(\mathrm{H}_{1}\right) \quad b \in\left(0, b^{*}\right]$ is a constant.

$\left(\mathrm{H}_{2}\right) f:[0,1] \times[0,+\infty) \rightarrow \mathbb{R}$ is continuous and

$$
f(t, x)+b x \geq 0
$$

Set

$$
G_{b}(t)=t^{\alpha-1} E_{\alpha, \alpha}\left(b t^{\alpha}\right)
$$

where

$$
E_{\alpha, \alpha}(x)=\sum_{k=0}^{+\infty} \frac{x^{k}}{\Gamma((k+1) \alpha)}
$$

is the Mittag-Leffler function (see $[1,2])$.

Next we consider the following boundary value problem:

$$
\left\{\begin{array}{l}
-D_{0+}^{\alpha} u(t)+b u(t)=f(t, u(t))+b u(t), \quad 0<t<1, \\
u(0)=0, \quad u(1)=\eta u(\xi)
\end{array}\right.
$$

It is clear that (1.1) is equivalent to (2.6). 
Set

$$
\begin{aligned}
& K_{0}(t, s)=\frac{1}{G_{b}(1)} \begin{cases}G_{b}(t) G_{b}(1-s), & 0 \leq t \leq s \leq 1, \\
G_{b}(t) G_{b}(1-s)-G_{b}(t-s) G_{b}(1), & 0 \leq s \leq t \leq 1\end{cases} \\
& q(s)=\frac{\eta K_{0}(\xi, s)}{G_{b}(1)-\eta G_{b}(\xi)} ; \\
& K(t, s)=K_{0}(t, s)+G_{b}(t) q(s) .
\end{aligned}
$$

Lemma 2.1 Suppose that $\left(\mathrm{H}_{1}\right)$ holds, and $y \in L[0,1]$. Then the unique solution of the problem

$$
\left\{\begin{array}{l}
-D_{0++}^{\alpha} u(t)+b u(t)=y(t), \quad 0<t<1 \\
u(0)=0, \quad u(1)=\eta u(\xi)
\end{array}\right.
$$

is

$$
u(t)=\int_{0}^{1} K(t, s) y(s) d s
$$

Proof By $[1,2]$, we know that the solution of (2.10) can be expressed by

$$
u(t)=-\int_{0}^{t} G_{b}(t-s) y(s) d s+c_{1} G_{b}(t)+c_{2} G_{b}^{\prime}(t) .
$$

By $u(0)=0$, we have $c_{2}=0$.

On the other hand, we have

$$
\begin{aligned}
& u(1)=-\int_{0}^{1} G_{b}(1-s) y(s) d s+c_{1} G_{b}(1), \\
& u(\xi)=-\int_{0}^{\xi} G_{b}(\xi-s) y(s) d s+c_{1} G_{b}(\xi) .
\end{aligned}
$$

Noting that $\eta \xi^{\alpha-1}=1$, and $0<\xi<1$, we have

$$
G_{b}(1)-\eta G_{b}(\xi)=\sum_{k=0}^{+\infty} \frac{b^{k}\left[1-\eta \xi^{(k+1) \alpha-1}\right]}{\Gamma((k+1) \alpha)}=\sum_{k=0}^{+\infty} \frac{b^{k}\left(1-\xi^{k \alpha}\right)}{\Gamma((k+1) \alpha)}>0
$$

Equations (2.11) and (2.12) yield

$$
c_{1}=\frac{\int_{0}^{1} G_{b}(1-s) y(s) d s-\eta \int_{0}^{\xi} G_{b}(\xi-s) y(s) d s}{G_{b}(1)-\eta G_{b}(\xi)} .
$$

Therefore, the solution of (2.10) is

$$
\begin{aligned}
u(t) & =-\int_{0}^{t} G_{b}(t-s) y(s) d s+\frac{\int_{0}^{1} G_{b}(1-s) y(s) d s-\eta \int_{0}^{\xi} G_{b}(\xi-s) y(s) d s}{G_{b}(1)-\eta G_{b}(\xi)} G_{b}(t) \\
& =\frac{\int_{0}^{1} G_{b}(t) G_{b}(1-s) y(s) d s-\int_{0}^{t} G_{b}(1) G_{b}(t-s) y(s) d s}{G_{b}(1)}
\end{aligned}
$$




$$
\begin{aligned}
& -\frac{\int_{0}^{1} G_{b}(1-s) y(s) d s}{G_{b}(1)} G_{b}(t)+\frac{\int_{0}^{1} G_{b}(1-s) y(s) d s}{G_{b}(1)-\eta G_{b}(\xi)} G_{b}(t) \\
& -\frac{\eta \int_{0}^{\xi} G_{b}(\xi-s) y(s) d s}{G_{b}(1)-\eta G_{b}(\xi)} G_{b}(t) \\
= & \int_{0}^{1} K_{0}(t, s) y(s) d s+\frac{\eta \int_{0}^{1} G_{b}(\xi) G_{b}(1-s) y(s) d s}{G_{b}(1)\left[G_{b}(1)-\eta G_{b}(\xi)\right]} G_{b}(t) \\
& -\frac{\eta \int_{0}^{\xi} G_{b}(1) G_{b}(\xi-s) y(s) d s}{G_{b}(1)\left[G_{b}(1)-\eta G_{b}(\xi)\right]} G_{b}(t) \\
= & \int_{0}^{1} K(t, s) y(s) d s .
\end{aligned}
$$

This completes the proof.

Lemma 2.2 Suppose that $\left(\mathrm{H}_{1}\right)$ holds. The function $K(t, s)$ has the following properties:

(1) $K(t, s)>0, \forall t, s \in(0,1)$;

(2) $\omega_{2}(s) t^{\alpha-1} \leq K(t, s) \leq \omega_{1}(s) t^{\alpha-1}, \forall t, s \in[0,1]$, where

$$
\omega_{1}(s)=G_{b}(1-s)+G_{b}(1) q(s), \quad \omega_{2}(s)=\frac{q(s)}{\Gamma(\alpha)} .
$$

Proof It is clear that we just need to prove that (2) holds.

By (2.4), we can get

$$
\begin{aligned}
\frac{t^{\alpha-1}}{\Gamma(\alpha)} & \leq G_{b}(t)=t^{\alpha-1} \sum_{k=0}^{+\infty} \frac{b^{k} t^{\alpha k}}{\Gamma((k+1) \alpha)} \leq t^{\alpha-1} G_{b}(1), \quad t \in[0,1] \\
G_{b}^{\prime}(t) & =\sum_{k=0}^{+\infty} \frac{b^{k} t^{(k+1) \alpha-2}}{\Gamma((k+1) \alpha-1)}>0, \quad t \in(0,1],
\end{aligned}
$$

and

$$
\begin{aligned}
G_{b}^{\prime \prime}(t) & =t^{\alpha-3}\left[\frac{\alpha-2}{\Gamma(\alpha-1)}+\sum_{k=1}^{+\infty} \frac{b^{k} t^{k \alpha}}{\Gamma((k+1) \alpha-2)}\right] \\
& =t^{\alpha-3} g\left(b t^{\alpha}\right)<t^{\alpha-3} g(b) \leq t^{\alpha-3} g\left(b^{*}\right)=0, \quad t \in(0,1),
\end{aligned}
$$

which implies $G_{b}(t)$ is strictly increasing on $[0,1]$, and $G_{b}^{\prime}(t)$ is strictly decreasing on $(0,1]$. By (2.15), we have

$$
\begin{aligned}
K(t, s) & =K_{0}(t, s)+G_{b}(t) q(s) \leq \frac{G_{b}(t) G_{b}(1-s)}{G_{b}(1)}+G_{b}(t) q(s) \\
& \leq G_{b}(1-s) t^{\alpha-1}+t^{\alpha-1} G_{b}(1) q(s)=\omega_{1}(s) t^{\alpha-1} .
\end{aligned}
$$

On the other hand, when $0<t \leq s<1$, noticing $G_{b}(0)=0$, and the monotonicity of $G_{b}(t)$, it is clear that

$$
G_{b}(t) G_{b}(1-s)>0 \text {. }
$$


When $0<s<t<1$, we have

$$
\begin{aligned}
\frac{\partial}{\partial s} & {\left[G_{b}(t) G_{b}(1-s)-G_{b}(t-s) G_{b}(1)\right] } \\
& =G_{b}^{\prime}(t-s) G_{b}(1)-G_{b}(t) G_{b}^{\prime}(1-s) \\
& \geq\left[G_{b}(1)-G_{b}(t)\right] G_{b}^{\prime}(1-s) .
\end{aligned}
$$

Integrating (2.20) with respect to $s$, we obtain

$$
\begin{aligned}
& G_{b}(t) G_{b}(1-s)-G_{b}(t-s) G_{b}(1) \\
& \quad \geq \int_{0}^{s}\left[G_{b}(1)-G_{b}(t)\right] G_{b}^{\prime}(1-\tau) d \tau \\
& \quad=\left[G_{b}(1)-G_{b}(t)\right]\left[G_{b}(1)-G_{b}(1-s)\right]>0 .
\end{aligned}
$$

By (2.7), (2.19), and (2.21), we get $K_{0}(t, s)>0, \forall t, s \in(0,1)$. Then

$$
\begin{aligned}
K(t, s) & =K_{0}(t, s)+G_{b}(t) q(s) \geq G_{b}(t) q(s) \\
& \geq \frac{t^{\alpha-1}}{\Gamma(\alpha)} q(s)=\omega_{2}(s) t^{\alpha-1}>0, \quad \forall t, s \in(0,1) .
\end{aligned}
$$

This completes the proof.

Let $E=C[0,1]$ be endowed with the maximum norm $\|u\|=\max _{0 \leq t \leq 1}|u(t)|, \theta$ is the zero element of $E, B_{r}=\{u \in E:\|u\|<r\}$. Define a cone $P$ by

$$
P=\{u \in E: u(t) \geq 0, t \in[0,1]\}
$$

Let

$$
\begin{aligned}
& A u(t)=\int_{0}^{1} K(t, s)[f(s, u(s))+b u(s)] d s . \\
& T u(t)=\int_{0}^{1} K(t, s) u(s) d s
\end{aligned}
$$

By means of Lemma 2.2 and the Arzela-Ascoli theorem, we can get $A: P \rightarrow P$ is completely continuous, $T: P \rightarrow P$ is completely continuous linear operator. By virtue of the Krein-Rutmann theorem and Lemma 2.2, we have the spectral radius $r(T)>0$ and $T$ has a positive eigenfunction corresponding to its first eigenvalue $\lambda_{1}=(r(T))^{-1}$. Since $\lambda=0$ is the eigenvalue of the linear problems (1.2), and $t^{\alpha-1}$ is the corresponding eigenfunction, we have the following lemma.

Lemma 2.3 Suppose that $\left(\mathrm{H}_{1}\right)$ holds, then the first eigenvalue of $T$ is $\lambda_{1}=b$, and $\varphi_{1}(t)=t^{\alpha-1}$ is the positive eigenfunction corresponding to $\lambda_{1}$, that is, $\varphi_{1}=b T \varphi_{1}$.

Lemma 2.4 ([27]) Let $P$ be a cone in a Banach space $E$, and $\Omega$ be a bounded open set in $E$. Suppose that $A: \bar{\Omega} \cap P \rightarrow P$ is a completely continuous operator. If there exists $u_{0} \in P$ with 
$u_{0} \neq \theta$ such that

$$
u-A u \neq \lambda u_{0}, \quad \forall \lambda \geq 0, x \in \partial \Omega \cap P,
$$

then $i(A, \Omega \cap P, P)=0$.

Lemma 2.5 ([27]) Let $P$ be a cone in a Banach space $E$, and $\Omega$ be a bounded open set in $E$. Suppose that $A: \bar{\Omega} \cap P \rightarrow P$ is a completely continuous operator. If

$$
A u \neq \lambda u, \quad \forall \lambda \geq 1, u \in \partial \Omega \cap P,
$$

then $i(A, \Omega \cap P, P)=1$.

\section{The uniqueness result}

Theorem 3.1 Assume that there exists $\lambda \in(0, b)$ such that

$$
|f(t, u)+b u-f(t, v)-b v| \leq \lambda|u-v|, \quad \text { for } t \in[0,1], u, v \in[0, \infty)
$$

then (1.1) has a unique nonnegative solution.

Proof Firstly, we will prove $A$ has fixed point in $P$.

Set

$$
Q=\left\{u \in P: \exists l_{1}, l_{2}>0 \text { such that } l_{1} t^{\alpha-1} \leq u(t) \leq l_{2} t^{\alpha-1}\right\} .
$$

For any $u \in P \backslash\{\theta\}$, let

$$
l_{i}(u)=\int_{0}^{1} \omega_{i}(s) u(s) d s, \quad i=1,2 .
$$

By Lemma 2.2, it is obvious that $l_{1}(u), l_{2}(u)>0$, and

$$
l_{1}(u) t^{\alpha-1} \leq(T u)(t) \leq l_{2}(u) t^{\alpha-1},
$$

that is,

$$
T: P \backslash\{\theta\} \rightarrow Q
$$

For any $u_{0} \in P \backslash\{\theta\}$, let

$$
u_{n}=A\left(u_{n-1}\right), \quad n=1,2, \ldots
$$

We may suppose that $u_{1}-u_{0} \neq \theta$ (otherwise, the proof is finished). Then there exists $l_{2}\left(\left|u_{1}-u_{0}\right|\right)>0$, such that

$$
T\left(\left|u_{1}-u_{0}\right|\right) \leq l_{2}\left(\left|u_{1}-u_{0}\right|\right) t^{\alpha-1}=l_{2}\left(\left|u_{1}-u_{0}\right|\right) \varphi_{1} .
$$


Thus

$$
\begin{aligned}
\left|u_{2}-u_{1}\right| & =\left|\int_{0}^{1} K(t, s)\left[f\left(s, u_{1}(s)\right)+b u_{1}(s)-f\left(s, u_{0}(s)\right)-b u_{0}(s)\right] d s\right| \\
& \leq \int_{0}^{1} K(t, s)\left|f\left(s, u_{1}(s)\right)+b u_{1}(s)-f\left(s, u_{0}(s)\right)-b u_{0}(s)\right| d s \\
& \leq \lambda \int_{0}^{1} K(t, s)\left|u_{1}(s)-u_{0}(s)\right| d s \\
& =\lambda T\left(\left|u_{1}-u_{0}\right|\right) \leq \lambda l_{2}\left(\left|u_{1}-u_{0}\right|\right) \varphi_{1}, \\
\left|u_{3}-u_{2}\right| & =\left|\int_{0}^{1} K(t, s)\left[f\left(s, u_{2}(s)\right)+b u_{2}(s)-f\left(s, u_{1}(s)\right)-b u_{1}(s)\right] d s\right| \\
& \leq \int_{0}^{1} K(t, s)\left|f\left(s, u_{2}(s)\right)+b u_{2}(s)-f\left(s, u_{1}(s)\right)-b u_{1}(s)\right| d s \\
& \leq \lambda \int_{0}^{1} K(t, s)\left|u_{2}(s)-u_{1}(s)\right| d s \\
& \leq \lambda^{2} l_{2}\left(\left|u_{1}-u_{0}\right|\right) T \varphi_{1}=\frac{\lambda^{2}}{b} l_{2}\left(\left|u_{1}-u_{0}\right|\right) \varphi_{1}, \\
& \ldots
\end{aligned}
$$

By induction, we can get

$$
\left|u_{n+1}-u_{n}\right| \leq\left(\frac{\lambda}{b}\right)^{n-1} \lambda l_{2}\left(\left|u_{1}-u_{0}\right|\right) \varphi_{1}
$$

Then, for any $n, m \in \mathbb{N}$, we have

$$
\begin{aligned}
\left|u_{n+m}-u_{m}\right| & \leq\left|u_{n+m}-u_{n+m-1}\right|+\cdots+\left|u_{n+1}-u_{n}\right| \\
& \leq\left[\left(\frac{\lambda}{b}\right)^{n+m-1}+\cdots+\left(\frac{\lambda}{b}\right)^{n}\right] \lambda l_{2}\left(\left|u_{1}-u_{0}\right|\right) \varphi_{1} \\
& \leq \frac{\left(\frac{\lambda}{b}\right)^{n}}{1-\frac{\lambda}{b}} \lambda l_{2}\left(\left|u_{1}-u_{0}\right|\right) \varphi_{1}=\frac{\lambda^{n+1} l_{2}\left(\left|u_{1}-u_{0}\right|\right)}{b^{n-1}(b-\lambda)} \varphi_{1} .
\end{aligned}
$$

So,

$$
\left\|u_{n+m}-u_{m}\right\| \leq \frac{\lambda^{n+1} l_{2}\left(\left|u_{1}-u_{0}\right|\right)}{b^{n-1}(b-\lambda)} \rightarrow 0, \quad n \rightarrow \infty
$$

which implies $\left\{u_{n}\right\}$ is a Cauchy sequence. Therefore, there exists a $u^{*} \in P$, such that $\left\{u_{n}\right\}$ converges to $u^{*}$. Clearly, $u^{*}$ is a fixed point of $A$.

In the following, we will prove the fixed point of $A$ is unique.

Suppose $v \neq u^{*}$ is a fixed point of $A$. Then there exists $l_{2}\left(\left|u^{*}-v\right|\right)>0$, such that

$$
T\left(\left|u^{*}-v\right|\right) \leq l_{2}\left(\left|u^{*}-v\right|\right) \varphi_{1}
$$


Then

$$
\begin{aligned}
\left|A u^{*}-A v\right| & =\left|\int_{0}^{1} K(t, s)\left[f\left(s, u^{*}(s)\right)+b u^{*}(s)-f(s, v(s))-b v(s)\right] d s\right| \\
& \leq \int_{0}^{1} K(t, s)\left|f\left(s, u^{*}(s)\right)+b u^{*}(s)-f(s, v(s))-b v(s)\right| d s \\
& \leq \lambda \int_{0}^{1} K(t, s)\left|u^{*}(s)-v(s)\right| d s \\
& =\lambda T\left(\left|u^{*}-v\right|\right) \leq \lambda l_{2}\left(\left|u^{*}-v\right|\right) \varphi_{1} .
\end{aligned}
$$

By induction, we can get

$$
\left|A^{n} u^{*}-A^{n} v\right| \leq\left(\frac{\lambda}{b}\right)^{n-1} \lambda l_{2}\left(\left|u^{*}-v\right|\right) \varphi_{1}
$$

So,

$$
\left\|u^{*}-v\right\|=\left\|A^{n} u^{*}-A^{n} v\right\| \leq\left(\frac{\lambda}{b}\right)^{n-1} \lambda l_{2}\left(\left|u^{*}-v\right|\right) \rightarrow 0, \quad n \rightarrow \infty .
$$

Consequently, the fixed point of $A$ is unique.

This completes the proof.

Remark 3.1 The unique nonnegative solution $u^{*}$ of (1.1) can be approximated by the iterative schemes: for any $u_{0} \in P \backslash\{\theta\}$, let

$$
u_{n}=A\left(u_{n-1}\right), \quad n=1,2, \ldots
$$

then $u_{n} \rightarrow u^{*}$.

Remark 3.2 If $f(t, 0) \equiv 0$ on $[0,1]$, then $\theta$ is the unique solution of $(1.1)$ in $P$; If $f(t, 0) \not \equiv 0$ on $[0,1]$, then the unique solution $u^{*}$ is a positive solution.

\section{Existence of positive solutions}

Theorem 4.1 Assume that $\left(\mathrm{H}_{1}\right),\left(\mathrm{H}_{2}\right)$, and the following assumptions hold:

$$
\begin{aligned}
& \liminf _{x \rightarrow 0+} \min _{t \in[0,1]} \frac{f(t, x)}{x}>0, \\
& \limsup _{x \rightarrow+\infty} \max _{t \in[0,1]} \frac{f(t, x)}{x}<0 .
\end{aligned}
$$

Then (1.1) has at least one positive solution.

Proof It follows from (4.1) that there exists $r_{1}>0$ such that

$$
f(t, x) \geq 0, \quad \forall(t, x) \in[0,1] \times\left[0, r_{1}\right] .
$$


Thus, for any $u \in \partial B_{r_{1}} \cap P$, we have

$$
A u(t)=\int_{0}^{1} K(t, s)[f(s, u(s))+b u(s)] d s \geq b T u(t)
$$

We may suppose that $A$ has no fixed points on $\partial B_{r_{1}} \cap P$ (otherwise, the proof is finished). Now we show that

$$
u-A u \neq \mu \varphi_{1}, \quad \forall u \in \partial B_{r_{1}} \cap P, \mu>0 .
$$

If otherwise, there exist $u_{1} \in \partial B_{r_{1}} \cap P$ and $\mu_{0}>0$ such that

$$
u_{1}-A u_{1}=\mu_{0} \varphi_{1}
$$

Then

$$
u_{1}=A u_{1}+\mu_{0} \varphi_{1} \geq \mu_{0} \varphi_{1}
$$

\section{Denote}

$$
\mu^{*}=\sup \left\{\mu: u_{1} \geq \mu \varphi_{1}\right\}
$$

It is clear that $\mu^{*} \geq \mu_{0}$ and $u_{1} \geq \mu^{*} \varphi_{1}$. Since $T(P) \subset P$, we have $b T u_{1} \geq \mu^{*} b T \varphi_{1}=\mu^{*} \varphi_{1}$. Then

$$
u_{1}=A u_{1}+\mu_{0} \varphi_{1} \geq b T u_{1}+\mu_{0} \varphi_{1} \geq\left(\mu^{*}+\mu_{0}\right) \varphi_{1}
$$

contradicts the definition of $\mu^{*}$. Hence (4.5) holds and we see from Lemma 2.4 that

$$
i\left(A, B_{r_{1}} \cap P, P\right)=0 .
$$

On the other hand, it follows from (4.2) that there exist $0<\sigma<1$ and $r_{2}>r_{1}$ such that

$$
f(t, x) \leq(\sigma-1) b x, \quad \forall t \in[0,1], x \geq r_{2} .
$$

Let $T_{1} u=\sigma b T u$. Then $T_{1}$ is a bounded linear operator and $T_{1}(P) \subset P$. Set

$$
W=\{u \in P \mid u=\mu A u, 0 \leq \mu \leq 1\} .
$$

In the following, we will prove that $W$ is bounded.

For any $u \in W$, set $\tilde{u}(t)=\min \left\{u(t), r_{2}\right\}$. Then

$$
f(t, u(t))+b u(t) \leq \sigma b u(t)+f(t, \tilde{u}(t))+b \tilde{u}(t) .
$$

Therefore,

$$
u(t)=\mu A u(t) \leq A u(t) \leq \sigma b T u(t)+A \tilde{u}(t) \leq T_{1} u(t)+M
$$


where

$$
M=\max _{(t, x) \in[0,1] \times\left[0, r_{2}\right]}\{f(t, x)+b x\} \int_{0}^{1} \omega_{1}(s) d s .
$$

Thus $\left(I-T_{1}\right) u(t) \leq M, t \in[0,1]$. Noticing $b$ is the first eigenvalue of $T$ and $0<\sigma<1$, we have $\left(r\left(T_{1}\right)\right)^{-1}=\sigma^{-1}>1$. So the inverse operator of $I-T_{1}$ exists, and

$$
\left(I-T_{1}\right)^{-1}=I+T_{1}+T_{1}^{2}+\cdots+T_{1}^{n}+\cdots .
$$

It follows from $T_{1}(P) \subset P$ that $\left(I-T_{1}\right)^{-1}(P) \subset P$. We have

$$
u(t) \leq\left(I-T_{1}\right)^{-1} M \leq M\left\|\left(I-T_{1}\right)^{-1}\right\|, \quad t \in[0,1],
$$

which implies $W$ is bounded.

Select $r_{3}>\max \left\{r_{2}, M\left\|\left(I-T_{1}\right)^{-1}\right\|\right\}$. Then by Lemma 2.5 , we have

$$
i\left(A, B_{r_{3}} \cap P, P\right)=1 .
$$

By (4.7) and (4.11), we have

$$
i\left(A,\left(B_{r_{3}} \backslash \bar{B}_{r_{1}}\right) \cap P, P\right)=i\left(A, B_{r_{3}} \cap P, P\right)-i\left(A, B_{r_{1}} \cap P, P\right)=1,
$$

which implies that $A$ has at least one fixed point on $\left(B_{r_{3}} \backslash \bar{B}_{r_{1}}\right) \cap P$. This means that BVP (1.1) has at least one positive solution.

This completes the proof.

Theorem 4.2 Assume that $\left(\mathrm{H}_{1}\right),\left(\mathrm{H}_{2}\right)$, and the following assumptions hold:

$$
\limsup _{x \rightarrow 0+} \max _{t \in[0,1]} \frac{f(t, x)}{x}<0
$$

and $f(t, 0) \not \equiv 0$ on $[0,1]$. Then (1.1) has at least one positive solution.

Proof It follows from (4.12) that there exists $r_{1}>0$ such that

$$
f(t, x) \leq 0, \quad \forall(t, x) \in[0,1] \times\left[0, r_{1}\right]
$$

Denote $T_{2} u=b T u$. Obviously, $r\left(T_{2}\right)=1$.

We may suppose that $A$ has no fixed points on $\partial B_{r_{1}} \cap P$ (otherwise, the proof is finished). In the following, we prove that

$$
A u \neq \mu u, \quad \forall u \in \partial B_{r_{1}} \cap P, \mu>1 .
$$

If otherwise, there exist $u_{1} \in \partial B_{r_{1}} \cap P, \mu_{0}>1$, such that $A u_{1}=\mu_{0} u_{1}$. It is clear that $\mu_{0} u_{1}=$ $A u_{1} \leq T_{2} u_{1}$, and

$$
\mu_{0}^{n} u_{1} \leq T_{2}^{n} u_{1}, \quad n=1,2, \ldots
$$


Therefore,

$$
\mu_{0}^{n} u_{1} \leq T_{2}^{n} u_{1} \leq\left\|T_{2}^{n}\right\|\left\|u_{1}\right\|
$$

Thus $r\left(T_{2}\right)=\lim _{n \rightarrow+\infty} \sqrt[n]{\left\|T_{2}^{n}\right\|} \geq \mu_{0}>1$, which contradicts $r\left(T_{2}\right)=1$. We have from Lemma 2.5

$$
i\left(A, B_{r_{1}} \cap P, P\right)=1 .
$$

Since $f(t, 0) \not \equiv 0$ on $[0,1]$, clearly we have $A \theta \neq \theta$, here $\theta$ is the zero element of $E$. So (4.15) implies that the problem (1.1) has at least one positive solution.

Remark 4.1 Suppose $u$ is a positive solution of (1.1), then there exist $l_{1}, l_{2}>0$, such that

$$
l_{1} t^{\alpha-1} \leq u(t) \leq l_{2} t^{\alpha-1}
$$

Example 4.1 (A 3-point boundary value problem at resonance) Consider the following problem:

$$
\left\{\begin{array}{l}
D_{0+}^{\frac{3}{2}} u(t)+f(t, u(t))=0, \quad 0<t<1, \\
u(0)=0, \quad u(1)=2 u\left(\frac{1}{4}\right) .
\end{array}\right.
$$

Since $\Gamma(\cdot)$ is strictly increasing on $[2,+\infty)$, for any $t \in[0,+\infty)$, we have

$$
\begin{aligned}
g(t) & =-\frac{1}{2 \sqrt{\pi}}+\sum_{k=1}^{+\infty} \frac{t^{k}}{\Gamma\left(\frac{3}{2} k-\frac{1}{2}\right)}=-\frac{1}{2 \sqrt{\pi}}+t+\sum_{k=2}^{+\infty} \frac{t^{k}}{\Gamma\left(\frac{3}{2} k-\frac{1}{2}\right)} \\
& \leq-\frac{1}{2 \sqrt{\pi}}+t+\sum_{k=2}^{+\infty} \frac{t^{k}}{\Gamma(k)}=-\frac{1}{2 \sqrt{\pi}}+t\left[1+\sum_{k=1}^{+\infty} \frac{t^{k}}{k !}\right] \\
& =-\frac{1}{2 \sqrt{\pi}}+t e^{t} .
\end{aligned}
$$

Noticing $\frac{1}{2 \sqrt{\pi}} \approx 0.282, \frac{1}{5} e^{\frac{1}{5}} \approx 0.243$, we have $g\left(\frac{1}{5}\right)<0$. Therefore $b^{*}>b_{1}:=\frac{1}{5}$.

Let

$$
f(t, x)= \begin{cases}\frac{1}{4}(1+t)(1-2 b) x, & (t, x) \in[0,1] \times[0,4), \\ \frac{1}{2}(1+t)(\sqrt{x}-b x), & (t, x) \in[0,1] \times[4,+\infty),\end{cases}
$$

where $b \in\left(0, b_{1}\right]$. It is clear that $\left(\mathrm{H}_{1}\right)$ and $\left(\mathrm{H}_{2}\right)$ hold. Moreover,

$$
\begin{aligned}
& \liminf _{u \rightarrow 0+} \min _{t \in[0,1]} \frac{f(t, u)}{u}=\frac{1-2 b}{4}>0, \\
& \limsup _{x \rightarrow+\infty} \max _{t \in[0,1]} \frac{f(t, x)}{x}=-\frac{b}{2}<0 .
\end{aligned}
$$

Therefore the assumptions of Theorem 4.1 are satisfied. Thus Theorem 4.1 ensures that (4.16) has at least one positive solution. 


\section{Competing interests}

The authors declare that they have no competing interests.

\section{Authors' contributions}

All authors contributed equally to the writing of this paper. All authors read and approved the final manuscript.

\section{Author details}

${ }^{1}$ School of Statistics, Qufu Normal University, Qufu, Shandong 273165, P.R. China. ${ }^{2}$ School of Mathematical Sciences, Qufu Normal University, Qufu, Shandong 273165, P.R. China. ${ }^{3}$ Department of Mathematics and Statistics, Curtin University, Perth, WA 6845, Australia.

\section{Acknowledgements}

The authors would like to thank the referee for his/her very important comments, which improved the results and the quality of the paper. This work was supported financially by the National Natural Science Foundation of China (11371221, 11571296), the Natural Science Foundation of Shandong Province of China (ZR2013AQ014, ZR2015AL002), the Specialized Research Fund for the Doctoral Program of Higher Education (20133705120003), and the Project of Shandong Province Higher Educational Science and Technology Program (J13LI08, J15LI16).

Received: 21 October 2016 Accepted: 16 December 2016 Published online: 05 January 2017

\section{References}

1. Podlubny, I: Fractional Differential Equations, Mathematics in Science and Engineering. Academic Press, New York (1999)

2. Kilbas, AA, Srivastava, HM, Trujillo, JJ: Theory and Applications of Differential Equations. Elsevier, Amsterdam (2006)

3. Su, X, Zhang, S: Unbounded solutions to a boundary value problem of fractional order on the half-line. Comput. Math. Appl. 61, 1079-1087 (2011)

4. Zhang, X, Wang, L, Sun, Q: Existence of positive solutions for a class of nonlinear fractional differential equations with integral boundary conditions and a parameter. Appl. Math. Comput. 226, 708-718 (2014)

5. Wang, Y, Liu, L, Wu, Y: Positive solutions for a class of fractional boundary value problem with changing sign nonlinearity. Nonlinear Anal. 74, 6434-6441 (2011)

6. Wang, Y, Liu, L, Wu, Y: Existence and uniqueness of a positive solution to singular fractional differential equations. Bound. Value Probl. 2012, 81 (2012)

7. Wang, Y, Liu, L, Wu, Y: Positive solutions for a nonlocal fractional differential equation. Nonlinear Anal. 74, 3599-3605 (2011)

8. Wang, Y, Liu, L: Necessary and sufficient condition for the existence of positive solution to singular fractional differential equations. Adv. Differ. Equ. 2015, 207 (2015)

9. Bai, Z: On positive solutions of a nonlocal fractional boundary value problem. Nonlinear Anal. 72, 916-924 (2010)

10. Zhang, $X, L i u, L, W u, Y$ : The uniqueness of positive solution for a fractional order model of turbulent flow in a porous medium. Appl. Math. Lett. 37, 26-33 (2014)

11. Zhang, X, Liu, L, Wu, Y, Wiwatanapataphee, B: Nontrivial solutions for a fractional advection dispersion equation in anomalous diffusion. Appl. Math. Lett. 66, 1-8 (2017)

12. Zhang, X, Liu, L, Wu, Y, Wiwatanapataphee, B: The spectral analysis for a singular fractional differential equation with a signed measure. Appl. Math. Comput. 257, 252-263 (2015)

13. Zhang, $X, W u, Y, L o u, C$ : Nonlocal fractional order differential equations with changing-sign singular perturbation. Appl. Math. Comput. 39, 6543-6552 (2015)

14. Zhang, $X$, Liu, L, Wu, Y: Variational structure and multiple solutions for a fractional advection-dispersion equation. Comput. Math. Appl. 68, 1794-1805 (2014)

15. Zhang, X, Liu, L, Wiwatanapataphee, B, Wu, Y: The eigenvalue for a class of singular $p$-Laplacian fractional differential equations involving the Riemann-Stieltjes integral boundary condition. Appl. Math. Comput. 235, 412-422 (2014)

16. Zhang, $X, L i u, L, W u, Y$ : The uniqueness of positive solution for a singular fractional differential system involving derivatives. Commun. Nonlinear Sci. Numer. Simul. 18, 1400-1409 (2013)

17. Zhang, X, Liu, L, Wiwatanapataphee, B, Wu, Y: Positive solutions of eigenvalue problems for a class of fractional differential equations with derivatives. Abstr. Appl. Anal. 2012, Article ID 512127 (2012)

18. Li, C, Luo, X, Zhou, Y: Existence of positive solutions of the boundary value problem for nonlinear fractional differential equations. Comput. Math. Appl. 59, 1363-1375 (2010)

19. Kosmatov, N: A boundary value problem of fractional order at resonance. Electron. J. Differ. Equ. 2010, 135 (2010)

20. Zhang, Y, Bai, Z, Feng, T: Existence results for a coupled system of nonlinear fractional three-point boundary value problems at resonance. Comput. Math. Appl. 61, 1032-1047 (2011)

21. Bai, Z: On solutions of some fractional $m$-point boundary value problems at resonance. Electron. J. Qual. Theory Differ. Equ. 2010, 37 (2010)

22. Bai, Z, Zhang, Y: Solvability of fractional three-point boundary value problems with nonlinear growth. Appl. Math Comput. 218, 1719-1725 (2011)

23. Infante, G, Zima, M: Positive solutions of multi-point boundary value problems at resonance. Nonlinear Anal. 69 , 2458-2465 (2008)

24. Yang, L, Shen, C: On the existence of positive solution for a kind of multi-point boundary value problem at resonance. Nonlinear Anal. 72, 4211-4220 (2010)

25. Wang, F, Cui, Y, Zhang, F: Existence of nonnegative solutions for second order $m$-point boundary value problems at resonance. Appl. Math. Comput. 217, 4849-4855 (2011)

26. Webb, J, Zima, M: Multiple positive solutions of resonant and non-resonant nonlocal boundary value problems. Nonlinear Anal. 71, 1369-1378 (2009)

27. Guo, D: Nonlinear Functional Analysis. Shandong Science and Technology Press, Jinan (1985) (in Chinese) 